\title{
Discontinuidad y autonomización en los trayectos vitales de los niños y jóvenes en situación de calle en La Paz y El Alto
}

Discontinuité et autonomisation des parcours de vie d'enfants et de jeunes en situation de rue à La Paz et El Alto

Discontinuity and Autonomization of the Life Courses of Children and Young

People in Street situations in La Paz and El Alto

\section{Robin Cavagnoud}

\section{OpenEdition}

Journals

Edición electrónica

URL: http://journals.openedition.org/bifea/7712

DOI: $10.4000 /$ bifea. 7712

ISSN: 2076-5827

Editor

Institut Français d'Études Andines

Edición impresa

Fecha de publicación: 1 diciembre 2015

Paginación: 463-479

ISSN: 0303-7495

Referencia electrónica

Robin Cavagnoud, « Discontinuidad y autonomización en los trayectos vitales de los niños y jóvenes en situación de calle en La Paz y El Alto », Bulletin de l'Institut français d'études andines [En línea], 44 (3) | 2015, Publicado el 08 diciembre 2015, consultado el 06 noviembre 2020. URL : http:// journals.openedition.org/bifea/7712 ; DOI : https://doi.org/10.4000/bifea.7712

\section{(c) (†) $\odot$}

Les contenus du Bulletin de l'Institut français d'études andines sont mis à disposition selon les termes de la licence Creative Commons Attribution - Pas d'Utilisation Commerciale - Pas de Modification 4.0 International. 
Bulletin de l'Institut Français d'Études Andines / 2015, 44 (3): 463-479

\title{
Discontinuidad y autonomización en los trayectos vitales de los niños y jóvenes en situación de calle en La Paz y El Alto
}

\section{Robin Cavagnoud*}

\begin{abstract}
Resumen
Los trayectos vitales de los niños y jóvenes en situación de gran precariedad están frecuentemente marcados por eventos que trastornan la organización de su vida cotidiana. Entre estos hechos, se puede mencionar la huida voluntaria o la expulsión forzada del hogar provocando una ruptura de los vínculos familiares y un modo de vida «de calle». A partir de los resultados de una investigación realizada en La Paz y El Alto, este artículo muestra en qué medida los trayectos vitales de los niños y jóvenes se caracterizan por procesos de individuación y fragmentación, los cuales indican el carácter ahora inoperante de las nociones de «carrera en la desviación» y de «pandilla» a favor de la idea de «red de entornos» mediante la cual los chicos circulan. Por último, la situación de los niños y jóvenes cuestiona las políticas de intervención hacia esta población, principalmente por parte de Organizaciones No Gubernamentales $(\mathrm{ONG})$ y otras asociaciones que logran integrarlos muy parcialmente en sus programas de apoyo.
\end{abstract}

Palabras clave: infancia, juventud, calle, familia, pobreza, trayectos vitales, O rganizaciones No Gubernamentales (ONG), Bolivia

* Socio-demógrafo, profesor investigador en el Departamento de Ciencias Sociales de la Pontificia Universidad Católica del Perú (PUCP), co-coordinador del grupo de investigación «Edades de la Vida y Educación» (EVE) e investigador afiliado al Instituto Francés de Estudios Andinos (IFEA, UMIFRE 17, MAEDI/CNRS). E-mail: rcavagnoud@ pucp.pe 


\title{
Discontinuité et autonomisation des parcours de vie d'enfants et de jeunes en situation de rue à La Paz et El Alto
}

\author{
Résumé
}

Les parcours de vie des enfants et des jeunes en situation de grande précarité sont fréquemment marqués par des événements qui bouleversent l'organisation de leur vie quotidienne. Parmi ces faits, on peut citer la fugue volontaire ou l'expulsion forcée du ménage entrainant une rupture des liens familiaux et un mode de vie "de rue ». À partir des résultats d'une enquête réalisée à La Paz et à El Alto, cet article montre dans quelle mesure les parcours de vie de ces enfants et de ces jeunes se caractérisent par des processus d'individuation et de fragmentation indiquant le caractère dorénavant inopérant des notions de «carrière dans la déviance » et de «bande » au profit de l'idée de « réseau d'entourages » dans lequel circulent les garçons. Enfin, la situation des enfants et des jeunes remet en question les politiques d'intervention auprès de cette population, principalement d'O rganisations non gouvernementales (ONG) et d'autres associations, qui ne parviennent que partiellement à les intégrer dans leurs programmes d'aide.

Mots-clés : enfance, jeunesse, rue, famille, pauvreté, parcours de vie, Organisations non gouvernementales (ONG), Bolivie

\section{Discontinuity and Autonomization of the Life Courses of Children and Young People in Street situations in La Paz and El Alto}

\begin{abstract}
The life courses of children and young people in precarious situations are frequently marked by events that disrupt the organization of their daily life. Among these facts, we can name voluntary running away or forced expulsion from household leading to family breakup and to a "street" lifestyle. Based on the results of fieldwork in La Paz and El Alto, this article shows how the life courses of these children and young people are characterized by individuation and fragmentation processes. This indicates a shift from inoperative notions of "career in deviance" and "band" in favor of the idea of "network of surroundings" where boys circulate. Finally, the situation of children and young people questions policy interventions with this population, mainly by non-Governmental Organizations (NGO) and associations, which manage only partially to integrate them into their support programs.
\end{abstract}

Keywords: Childhood, Street, Family, Poverty Life Courses, Non Gouvernamental O rganizations (NGO), Bolivia

\section{INTRODUCCIÓN}

En Bolivia como en otros países en vía de desarrollo, los trayectos vitales de los niños y jóvenes en situación de gran precariedad están frecuentemente marcados por eventosque trastornan la organización de su vida cotidiana. Entre estoshechos, se puede mencionar el abandono, el fallecimiento o la migración de un padre, la pérdida repentina de un empleo o la huida voluntaria y la expulsión forzada del 
hogar, los cuales provocan una fractura de los vínculos familiares y un modo de vida en situación de calle. Estos cambios decisivos acarrean «rupturas biográficas» (Bessin et al. , 2010) y una recomposición de los espacios de vida y de los «entornos» de los niños (Golaz, 2009). En el caso de los niños que escapan de su hogar como consecuencia de las formas de violencia físicas y psicológicas, la ruptura familiar conlleva a un abandono del sistema escolar y la integración a grupos marginales de niños y jóvenes, organizados en torno a prácticas desviantes como el robo 0 el consumo de alcohol e inhalantes (Cavagnoud, 2014). En el caso de La Paz y El Alto, si bien el modo de vida de los niños «en la calle» se caracterizaba durante los años 1990 e inicios de los años 2000 por la pertenencia a una «pandilla» con una organización interna, relaciones horizontales y de jerarquía de poder entre grupos de niños y jóvenes (Suremain, 2006), una investigación de campo realizada entre 2010 y 2011 enseñó, por el contratio, trayectos vitales mayormente orientados hacia tomas de decisión individuales y proyectos que no dependen del grupo de pares. Esta evolución hacia una individuación de los itinerarios sociales de los niños y jóvenes en situación de calle rompe con la mayoría de los análisis sobre esta problemática desde los años 1980 en las metrópolis latinoamericanas como Montevideo (Lucchini, 1996) y México (Pérez López, 2009), en particular con respecto a la función de grupos de pares en la calle (Taracena \& Tavera, 2005) y el funcionamiento de las pandillas sobre la base de vínculos afectivos alternativos a la familia (M érienne Sierra, 1994; Suremain, 2006; 2013).

En dos artículos recientes (Cavagnoud, en prensa $a ; b$ ), se ha demostrado que la noción de supervivencia no corresponde a un uso sistemático para caracterizar el modo de vida de los niños y jóvenes en situación de calle y cómo estos se apoyan de forma temporal en «soportes» (Castel \& H aroche, 2001) como el grupo de pares, el medio institucional y el trabajo para atenuar situaciones de gran precariedad. El presente artículo se interesa en demostrar la individuación y fragmentación de los trayectos vitales de los niños y jóvenes en situación de calle en el caso de La Paz y El Alto. En la medida en que las nociones de «carrera en la desviación»o de «pandilla» no reflejan los resultados de la investigación, sería más apropiado referirse a la idea de «red de entornos» mediante la cual los niños van circulando. Por último, esta situación actual de los niños y jóvenes cuestiona las políticas de intervención con esta población, principalmente por parte de O rganizaciones No Gubernamentales (ONG) y del medio asociativo y humanitario, que logran muy parcialmente integrarlos de forma duradera en sus programas de ayuda.

\section{ANALIZAR LA SITUATION DE LA INFANCIA EN UN CONTEXTO DE PRECARIEDAD URBANA}

\section{1. Pobreza y precariedad en La Paz y El Alto}

La Paz es la sede del gobierno y la capital política de Bolivia. U bicado en un valle a 3600 metros de altura, esta ciudad se encuentra muy poblada en las laderas de las montañas que marcan su extensión geográfica y sus límites en términos de 
capacidades de acogida de nuevas familias procedentes de los campos andinos. A pesar de la creciente ocupación de terrenos ubicados en los márgenes urbanos de la Zona Sur de La Paz, la ciudad limítrofe de El Alto es aquella que concentra el crecimiento demográfico del continuum urbano de ambas ciudades. Esta se extiende en el Altiplano a unos 4000 metros de altura. Dispone de amplios espacios para la instalación de migrantes o la construcción de nuevos domicilios para alojar a las jóvenes generaciones adultas (Poupeau, 2011). Entre los años 2001 y 2012, la población total de El Alto aumentó en un 29,8\% pasando de 649958 a 843934 habitantes mientras que la población de La Paz disminuyó en un 4,1\% pasando de 793293 a 764617 habitantes (INE, 2001; 2012). Este crecimiento demográfico resultó más intenso en la ciudad de El Alto que al nivel nacional $(21,2 \%$ entre ambas fechas) y tuvo como efecto ubicar este antiguo barrio marginal de La Paz en el segundo rango de las ciudades más pobladas del país detrás de Santa Cruz que cuenta 1453549 habitantes (INE, 2001; 2012). Si bien la población total del conjunto urbano de La Paz y El Alto supera el de Santa Cruz, la característica que diferencia la metrópolis del Altiplano con la de las tierras bajas, es la proporción de la población en situación de pobreza: 66\% para el departamento de La Paz (incluyendo El Alto) y 38\% para el de Santa Cruz, el más bajo del país (INE, 2011). La ciudad de El Alto muestra por otro lado un Índice de Desarrollo H umano (ID H) más débil que las dos otras ciudades: 0,661 contra 0,730 para La Paz y 0,765 para Santa Cruz (PNUD, 2005).

A pesar de la emergencia de una fuerte movilización política y asociativa (Lazar, 2013) y de un dinamismo comercial conectado con la globalización (Tassi et al., 2013), El Alto presenta numerosos señales de precariedad con respecto al mercado laboral con una mayoría de la Población Económicamente Activa (PEA) sin contrato de trabajo alguno, en particular para las jóvenes generaciones (Yapu \& Q uisbert, 2008). D e igual manera, se pueden mencionar las carencias relacionadas con el sistema de cuidado y de salud, de educación pública y de acceso a los servicios básicos como el abastecimiento de agua potable (Poupeau, 2009; 2013) así como problemas de violencia doméstica y escolar (Mollericona et al., 2011) y servicios de transporte urbano congestionados y contaminantes.

En este contexto de precariedad generalizada en la ciudad, ha ido apareciendo desde hace unos 20 años grupos de niños y jóvenes que viven en el espacio público, al margen de su familia y de la escuela. En la metrópolis de La Paz y El Alto, se estiman unos 1000 niños y jóvenes en situación de calle. Se trata de una población muy variable según las entradas y salidas de la familia, las estadías en los hogares o la cárcel (Cavagnoud, 2014). Más allá de la cantidad efectiva de niños «en la calle», resulta más interesante enfocarse en los trayectos vitales, la imposición de cifras acarreando el riesgo de «neutralizar» las situaciones heterogéneas de los niños y jóvenes, y de simplificar e incluso hacer invisibles sus modos de vida caracterizados por una fuerte discontinuidad y diversidad de espacios de socialización. En la literatura científica sobre la infancia y la calle, pocos trabajos han adoptado un enfoque a partir de los trayectos vitales. No obstante, se puede citar el de Pérez López (2009) quien, a partir de una aproximación biográfica, 
identifica las fases de continuidad por el trabajo y la integración en un grupo de pares que proporcionan cierta estabilidad a los niños y jóvenes en su vida cotidiana. Pérez López especifica que solo un enfoque dinámico que descansa en la historia de cada niño nos puede ayudar para entender su modo de vida y la evolución de su posición en su universo social y afectivo.

\section{2. Aspectos metodológicos y población estudiada}

Para estudiar la situación de los niños y jóvenes en situación de calle, el enfoque biográfico resulta ser el más apropiado pues permite identificar todas las etapas, desde el nacimiento hasta el día de la entrevista, que fijan periodos a lo largo de sus trayectos vitales. Contribuye también en explicar las transiciones entre estas etapas a partir de los eventos que producen rupturas y recomposiciones en su historia personal. El enfoque que proponemos lleva por lo tanto a una reflexión sobre el conjunto de los trayectos vitales de los niños y jóvenes, distinguiendo las mayores secuencias que estructuran su trayectoria.

El presente artículo se apoya en una investigación realizada en las metrópolis de La Paz y de El Alto entre septiembre de 2010 y julio de 2011 en una muestra no probabilística de 27 niños y jóvenes de 10 a 31 años en situación de calle, actual o pasada. Estos fueron encontrados en el espacio público o en una de las instituciones que trabajan con esta población. Nuestro material empírico está constituido por relatos de vida que recuerdan en detalle la historia de cada niño y joven desde su nacimiento así como de observaciones etnográficas con grupos que se encuentran en la calle (campos de fútbol, puentes, entradas de mercado). Por lo tanto la singularidad de esta investigación se basa en los trayectos vitales de niños que están en situación de calle o de jóvenes que han estado en situación de calle durante un periodo significativo de su infancia. Este enfoque cualitativo permite identificar los eventos que han acarreado bifurcaciones en su itinerario personal así como observar a posteriori las fases de recomposición, en respuesta a sus iniciativas o de forma independiente a su voluntad.

En esta perspectiva, los datos de la investigación han sido analizados con ayuda de la ficha Ageven, una herramienta que permite ubicar los eventos que ocurren a lo largo de la historia de vida de un individuo (niño ego en este caso) y evidencia la relación entre estos hechos importantes. El uso de esta herramienta privilegia un enfoque dinámico y ayuda a distinguir las situaciones de ruptura y los cambios tanto de residencia, de actividades como de espacios de socialización según una sucesión de secuencias con causas y desafíos específicos. De hecho, permite tomar en cuenta el encadenamiento de experiencias en el plano factual, las cuales llevan a los actores hacia posiciones y estatus diferentes que modifican tanto su estado subjetivo, sus intereses, perspectivas como oportunidades. Todo evento que implica una bifurcación o «accidente biográfico» lleva efectivamente una dimensión a la vez objetiva a partir de la ocurrencia de hechos específicos, y subjetiva por lo que significan íntimamente para los individuos (N égroni, 2010). Marca el punto de partida en una nueva secuencia de vida cuyos encadenamientos 
muestran divisiones temporales (Lelièvre $\&$ Vivier, 2001). A partir del relato de vida y de la edad como unidad de análisis funcional (Lelièvre \& Vivier, 2001: 1054), la ficha Ageven contribuye a un estudio prosoprográfico de la dinámica interna de cada secuencia entre los espacios de socialización de los individuos y de sus múltiples trayectorias (familiares, escolares, profesionales, etc.). Para ello, coloca el conjunto de elementos referidos al trayecto de los actores en una matriz explicativa amplia que deja un espacio importante a todos los campos que cuentan en la vida de los niños y que pueden mostrar una evolución en el transcurso de su existencia: la familia, la escuela, el trabajo, el grupo de pares, etc. Los datos biográficos están informados de manera datada, descrita y interrelacionada en un calendario único centrado en una temporalidad individual que se articula con las dinámicas familiares, escolares y sociales cuyas retribuciones - afectivas, educativas, financieras, etc.- son variables en el tiempo.

\section{UNA FRAGMENTACIÓ N DE LOS TRAYECTOS VITALES DE LOS NIÑOS Y JÓ VENES}

Entre los 27 casos de niños y jóvenes en situación de calle reunidos a lo largo de la investigación, se nota en primer lugar una gran singularidad de los trayectos vitales remitiendo a una heterogeneidad en la cronología de los accidentes biográficos que marcan la existencia de los niños. Ello se refiere particularmente a los efectos de la violencia en la familia, la integración progresiva en el universo de la calle y la decisión de dejar el hogar para transitar hacia un modo de vida que excluye las relaciones familiares. No obstante, en el estudio de las historias de vida facilitado por la ficha Ageven, el análisis prosoprográfico nos ayuda a distinguir tres secuencias mayores; la primera caracterizada por la pequeña infancia en la familia (T1), la secunda marcada por la huida y luego la situación de calle (T2), y la tercera mostrando una circulación entre varios entornos en los cuales el medio institucional, la calle, idas y vueltas en la familia, además de pasos puntuales por la cárcel o el compromiso en el servicio militar (T3) (Cavagnoud, en prensa a). O tras secuencias pueden intercalarse, por ejemplo un periodo de fuerte inestabilidad familiar entre la pequeña infancia y la entrada en un modo de vida de calle.

\section{1. El ejemplo de Wilson}

Wilson nació y creció en La Paz con sus padres y cinco hermanos (ocupa la tercera posición en su hermandad). Uno de sus hermanos falleció un año después de su nacimiento mientras que el mayor fugó del domicilio familiar para vivir en la calle cuando Wilson tenía 3 años, dándole una forma de ejemplo a seguir más adelante. El hogar se caracterizó durante su infancia por una precariedad en el tipo de empleo de los padres (padre albañil y madre vendedora ambulante), sin ingresos suficientes para enfrentarse con las necesidades de todos los hijos. A ello se añadió el alcoholismo del padre y la recurrencia de las escenas de violencia 
presenciadas por Wilson, lo cual agudizó un ambiente de profundo malestar en la familia (secuencia T1). Wilson se dio a la fuga del domicilio familiar a la edad de 7 años y fue recogido por educadores de la asociación Alalay que lo derivaron a un centro cerrado donde se quedó casi tres años estudiando de forma continua (secuencia T2). Algunos problemas de entendimiento con los educadores lo incentivaron después a escaparse a la calle donde se quedó hasta el día de la entrevista, a pesar de pasos regulares en instituciones (para curarse de problemas de salud), unas estadías en la cárcel para menores de La Paz («El Trono») así como regresos esporádicos en la familia (secuencia T3).

Durante sus primeros años en la calle entre los 10 y 14 años, Wilson compartió su tiempo entre la mendicidad, el robo y el consumo de inhalantes (pegamento y quitaesmalte) con su grupo de pares en un área poco concurrida de La Paz, ubicada debajo del puente de Las Américas. Si bien regresó a instituciones conocidas gracias a sus contactos con educadores y donde no solía permanecer más de unas semanas, Wilson se dedicó exclusivamente a robar en el centro de La Paz a partir de los 14 años, exponiéndole tanto a la policía como a los transeúntes quienes a veces no dudan en aplicar formas de justicia comunitaria. Su mayor dedicación al robo en las calles de La Paz coincidió con su creciente consumo de alcohol («aguardiente») como práctica rutinaria antes de acudir al espacio público para robar y, después de esta actividad, durante sus salidas nocturnas. Wilson vivió en aquel entonces parcialmente con sus amigos, con quienes consumía alcohol (en abundancia), pero sobretodo dormía en cuartos de hostales mientras mantenía vínculos irregulares con su familia. A los 14 años, se encontró por casualidad con su hermano en la calle. Este evento le provocó mucha emoción aunque no lo animó a volver a instalarse de nuevo en el domicilio familiar. Unos meses después, su hermano terminó convenciéndolo pero esta tentativa fracasó al cabo de unos días por las malas relaciones de Wilson con su padre. Durante la adolescencia, este chico acumuló varias estadías de unas semanas o meses en diferentes hogares de acogida y proyectos sociales como la M isión Adulam, Alalay, Maya Paya Kimsa, la Casa de Paso y luego Luz de Esperanza donde lo hemos conocido y donde se quedó unos meses antes de volver a la calle en la zona del cementerio municipal de La Paz. Lo hemos vuelto a encontrar en diferentes oportunidades o en la calle o en el centro Luz de Esperanza para bañarse, alimentarse, jugar fútbol y lavar su ropa una vez a la semana.

\section{2. Eventos y distribución de los trayectos vitales en secuencias}

El ejemplo de Wilson es representativo de la muestra de niños y jóvenes en situación de calle. Su trayecto vital se descompone en varias secuencias y una sucesión de espacios de socialización que se vuelven a identificar con unos matices entre uno caso y otro, en las cuales la calle no es un centro de gravedad continuo. La secuencia T1 de la pequeña infancia se inicia en el nacimiento y termina en el momento de la huida siendo esta la primera situación de ruptura que acarrea la transición de los niños hacia responsabilidades y un estatus al margen de los 
vínculos familiares. Esta secuencia es portadora de una dinámica de inestabilidad y ruptura que se caracteriza, en todos los casos, por una recurrencia de la violencia doméstica relacionada con los problemas de alcoholismo del padre, reforzada por la falta de apoyo exterior a la familia nuclear, en particular a través de los abuelos (muchos de ellos radican en el campo), tíos o instituciones especializadas en la protección social de la pequeña infancia. La situación de pobreza en el hogar con padres trabajando en actividades del subempleo con escasos ingresos se impone también como obstáculo al bienestar familiar, agregando frustración a los actos de violencia ya evidenciados. Al final de esta primera secuencia, los niños suelen tener un contacto inicial con el mundo de la calle, en particular cuando uno de los amigos o hermanos mayores, como en el ejemplo de Wilson, les da la oportunidad de socializarse con niños en ruptura de su familia. La escolarización se interrumpe en el momento en que los niños dejan su familia para desplazarse a un modo de vida «de calle», lo cual marca el inicio de la secuencia T2. En el caso de Wilson, se nota un matiz al respecto en la medida en que este niño conoció primero un periodo de cerca de tres años de institucionalización a raíz de una fase muy corta de unos días en la calle después de escaparse de la violencia en su familia. Se trata aquí de uno de los pocos ejemplos en los cuales mecanismos de protección social se implementan para intervenir hacia la infancia «en peligro» desde los primeros días en la calle. En la mayoría de los casos, el paso de los niños en la secuencia T2 se produce a raíz de su huida voluntaria de la familia para vivir de forma duradera en el seno de un grupo de pares en espacios de la ciudad poco concurridos y alejados de los sectores residenciales (debajo de los puentes en espacios llamados «torrentes» 0 en terrenos sin habitaciones cercanas). A raíz de esta decisión, los niños suelen experimentar una impresión de alivio así como un sentimiento de libertad por el hecho de dejar atrás la violencia doméstica de la cual fueron víctimas y de encontrar un espacio de recursos dentro de un grupo protector en la calle.

Gonzalo (21 años de edad, alternancia entre la familia y la calle entre los 6 y 15 años):

La calle la he conocido cuando tenía 7 años ya, cuando he estado abajo en la terminal. De la terminal me iba a dormir ahí, en la calle y ahí había para cuidar autos, estacionamientos y ahí cuidaba autos. (...) En mi familia ya no me querían, a veces me decían «ya bájate no más a la calle, qué estás haciendo aquí». Me gustaba más la calle, ya no me gustaba ya mi casa. Porque ya estaba con mis amigos y creo que nos entendíamos bien... Reíamos en la calle, hacía mis cosas, locuras, todo hacía... pero mi casa ya no me gustaba (Luz de Esperanza, 8 de diciembre de 2010).

La mayoría de los niños se dedican a actividades como el lustrado de botas, la venta ambulatoria, el cuidado de carros o la cobranza de pasajes en los microbuses. Su trabajo no representa un campo en sí, sino un recurso a la vez económico, social y simbólico para atenuar la falta de dinero, cubrir sus necesidades alimentarias y encontrar un rol y función que cumplir en el espacio público, encontrándose ahora fuera de la familia y de la escuela. 


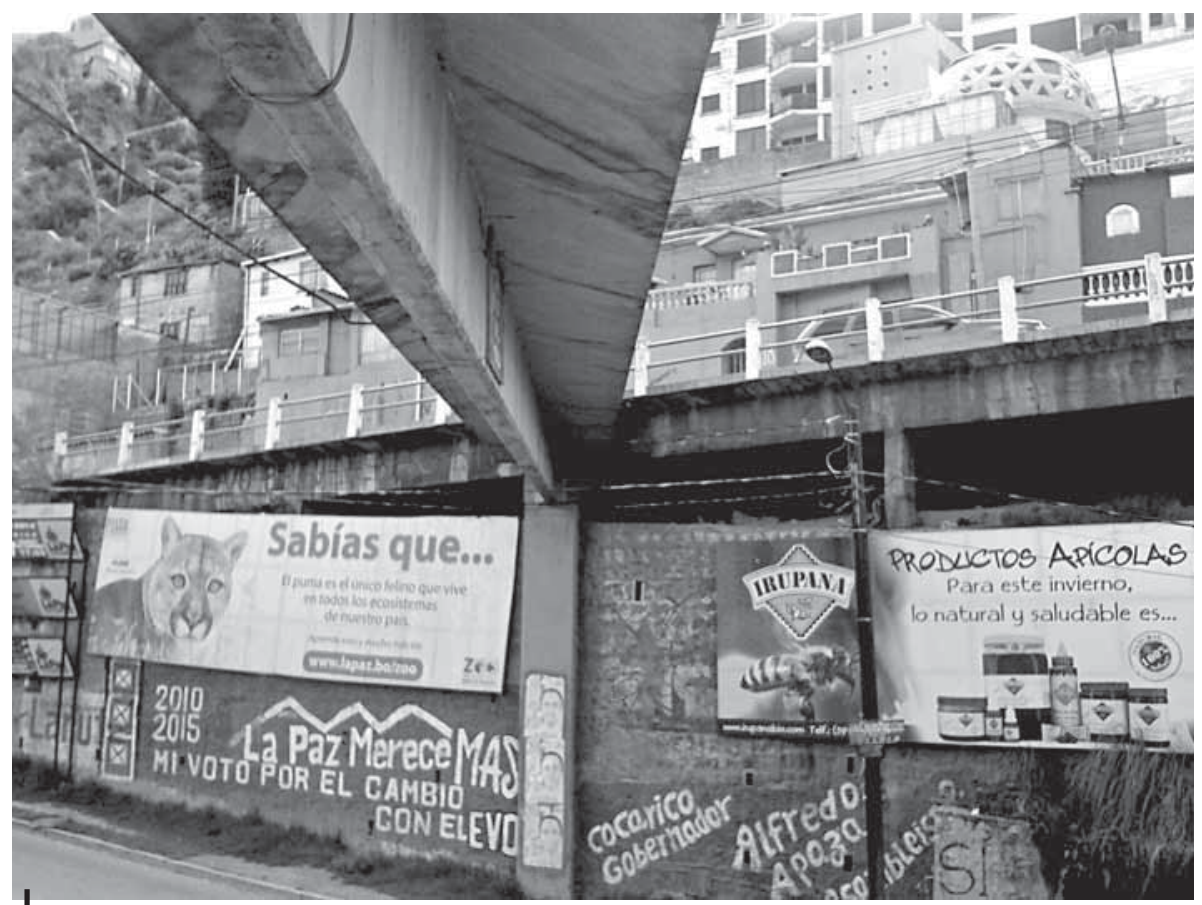

Figura 1 - Ejemplo de «torrentes» debajo de un puente en La Paz

(c) RC $30 / 03 / 2011$

A diferencia de los testimonios de jóvenes de unos treinta años que formaban parte de grupos de niños durante los años 1990, las agrupaciones de niños tienden a reducirse en el tiempo y a desagregarse más fácilmente de acuerdo a los proyectos de cada uno y a las formas de violencia que se ejercen en los grupos de pares 0 al contacto con la policía. La secuencia T2 tiende así a desarrollarse en poco tiempo (unos meses como máximo) para dejar lugar a una secuencia de vida (T3) en la cual los niños muestran una importante movilidad entre la calle que encuentran según sus deseos de socialización (reencontrarse con amigos), el entorno de los hogares que les puede servir de soporte en caso de necesidad urgente (curarse de una herida, esconderse después de una agresión, lavar su ropa, comer), la familia y en particular los miembros de su hermandad, el medio carcelario (por ejemplo Wilson cuenta con un total de 16 estadías en la cárcel de menores de La Paz) y eventualmente la escuela para aquellos que logran volver a escolarizarse. Algunos recurren también a la migración para un nuevo proyecto de vida mientras que otros adolescentes se comprometen desde los 16 años en el ejército para cumplir su servicio militar.

La secuencia T3 se ha impuesto para las actuales generaciones de niños y jóvenes en situación de calle como el periodo central y más largo. Se caracteriza por una sucesión de desercionesy reinsercionesasí como por una importante discontinuidad en los trayectos vitales de los actores. El acortamiento de la fase propiamente dicho 
«en la calle» deja lugar a un encadenamiento y superposición de campos sociales a lo largo de la historia personal entre los cuales algunos se oponen (la calle y el medio institucional) y otros se complementan (la familia, la escuela o el trabajo por ejemplo). Si bien en la secuencia T2, los niños comparten experiencias cotidianas similares a través de actividades rutinarias construidas sobre vínculos de solidaridad y ayuda mutua, o el consumo de alcohol, esta estabilidad relativa desaparece en la secuencia T3 para dar origen a una circulación individual entre campos sociales que presentan oportunidades e intereses específicos, en los cuales cada niño ocupa una posición singular. El caso de W ilson demuestra en qué medida esta secuencia se alarga en el tiempo (13 años al menos en este ejemplo) y se caracteriza por un doble movimiento a la vez de continuidad (mantenimiento de los vínculos con la calle, los hogares y la familia) y de cambio (idas y vuelta entre estos campos) de acuerdo a las circunstancias (peleas, heridas), a los recursos (necesidad de robar 0 trabajar en la calle), de las coacciones (expulsión de un hogar o imposibilidad de permanecer ahí en pareja) que dependen tanto de determinismos exteriores como de la misma voluntad de los niños como agentes sociales dotados de una clara capacidad de decisiones en el curso de su vida cotidiana.

\section{UNA INDIVIDUACIÓ N EN LA RELACION CON LA CALLE}

\section{1. Una pluralidad de espacios y roles sociales}

Las evoluciones observadas del fenómeno de los niños y jóvenes en situación de calle en la metrópolis de La Paz y El Alto testifican una ausencia de «carrera en la calle» (Lucchini, 1993) que se reflejaría por una identidad desviante traducida por una posición y auto representación como «niño de la calle». En la secuencia T3, los niños y jóvenes combinan y yuxtaponen una pluralidad de roles sociales en función de los espacios donde se encuentran sucesivamente: el de «par» 0 «amigo» cuando están en grupo en la calle para pasar el tiempo, jugar o consumir alcohol (también se llaman «hermanos» entre ellos), el de «niño» en búsqueda de protección durante los pasos en las institución de ayuda, el de «trabajador» en la calle (como categoría amplia que incluye los robos) o el de niño, hijo o hermano durante las visitas o estadías en su familia. De hecho, ya no es pertinente en la actualidad referirse a la constitución de «pandillas» de niños como el pasado cuando «Los Solitarios» y «Los Intocables» entraban en conflicto en las calles de la ciudad según relaciones jerárquicas basadas en la edad (Suremain, 2006).

Según los resultados de la investigación, la situación actual de los niños y jóvenes se aparenta más a la de una «red de entornos» mediante la cual circulan según los espacios de socialización a su disposición, de acuerdo a sus proyectos y lógicas individualizadas para buscar una forma de estabilidad y escaparse de la supervivencia. La sucesión en las estadías más o menos prolongadas entre los diferentes espacios de vida - calle, instituciones, familia, migración, servicio militar - revela tácticas de adaptación por parte de los niños y jóvenes con miras a la mejor inversión posible de los recursos - emocionales, afectivos, 


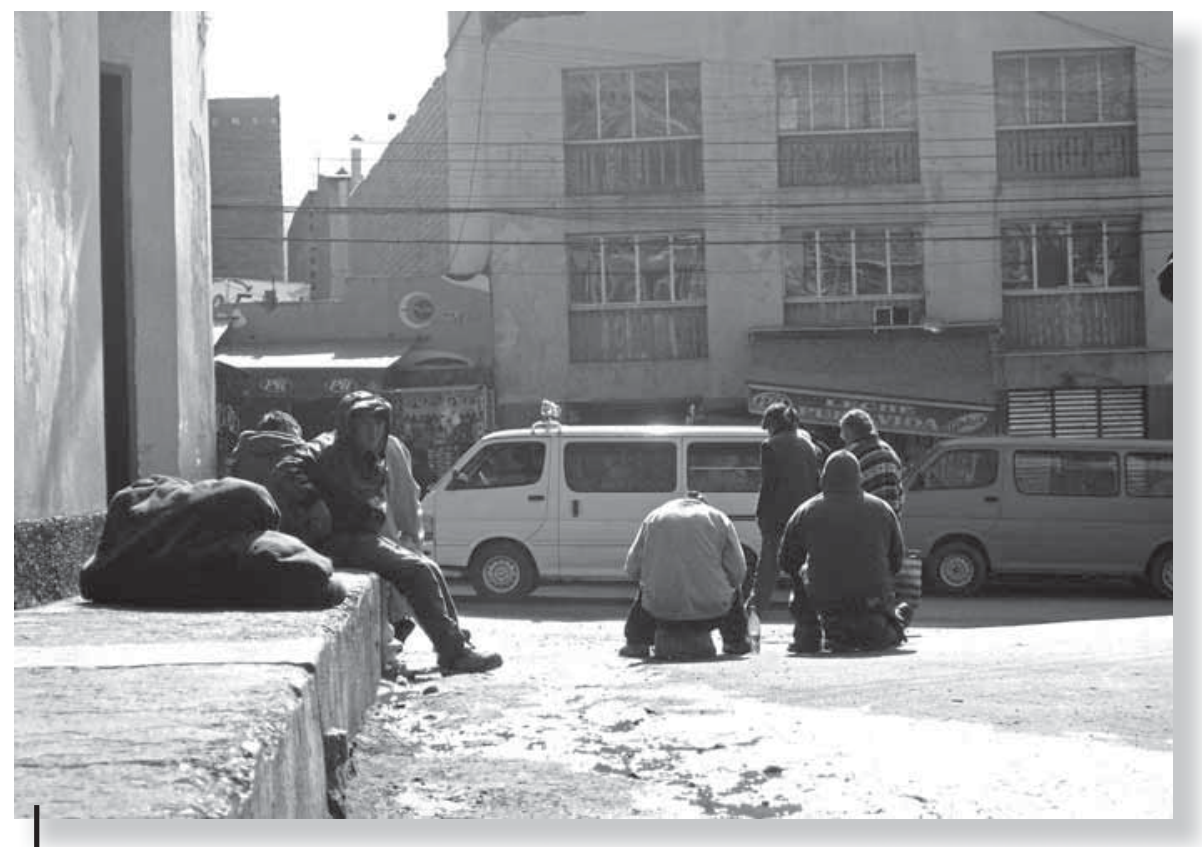

Figura 2 - Jóvenes reunidos cerca del cementerio municipal de La Paz

(c) RC, $22 / 06 / 2011$

materiales, identidarios y sociales- en los distintos momentos de su trayecto. En este sentido, representan un quiebre con los vínculos tradicionales basados en formas de dependencia comunitaria y familiar, así como el paso de un actor encastrado en un todo social estructurado a un individuo que se posiciona frente a una variedad de opciones revelando un enraizamiento problemático en la vida social. Su capacidad de modificación de la vida cotidiana y las decisiones de colocación entre los entornos a partir de sus intenciones, estrategias y de una interpretación constante de los factores que los rodean, reflejan una afirmación de su subjetividad, una conciencia de sí mismo como sujetos y una «libertad fundadora» propia de la modernidad (Elias, 1987; Martuccelli, 2002).

Desde el nacimiento, la situación de los niños y jóvenes es atravesada por la dominación social, primero con respecto a la pobreza que afecta su familia durante la infancia y luego, después de la ruptura de los vínculos familiares, en su dificultad de reconocimiento como sujetos, según una deslegitimación de su estatus de niño y frente a la violencia que conocen (por la policía o dentro de los grupos de pares). Es con el objetivo de contrapesar estas formas de dominación económica, social y simbólica que los niños y jóvenes afirman una individualidad expresada en su paso entre los campos sociales según estrategias a la vez de evasión y de captación de legitimidad a lo largo de su trayecto. En este contexto, la calle no aparece como un espacio de vida autónomo en el largo plazo sino que se combina con otros espacios cuyos pasos corresponden a aspiraciones individuales de salida y llegada 
según las limitaciones situacionales que conducen a los niños hacia «bifurcaciones activas» (H élardot, 2006), es decir decisiones tomadas «en un momento preciso e identificable a posteriori [afin] de romper la situación estable [vivida] actualmente y [tomar] la iniciativa del cambio» (p. 1650).

Lhonny (16 años, alternancia entre la calle, la familia, el medio institucional desde la edad de 11 años):

Yo últimamente he elegido estar mejor en un hogar porque en la calle muriéndome de frío, más bien aquí tengo una cama, tengo comida, tengo una ropa con que vestirme. Entonces he elegido venir aquí porque como he estado en hogares he dicho, sí me voy a ir a un hogar y yo sé que voy a estar bien en un hogar y... porque estaba temblando, me hacía temblar el trago. Pero también quiero encontrar a mi mamá, la quiero ver para que vea que estoy bien. Tengo otros amigos que vivían en Villa Fátima. Me han dicho que a mi mamá ya no la han visto vender, solo se han enterado que mi mamá estaba mal, ha estado en el hospital y yo no sabía nada de eso. Q uiero verla para ayudarla (Luz de Esperanza, 21 de enero de 2011).

Las idas y vueltas frecuentes en la calle reflejan elecciones relacionadas con los conflictos con los educadores o con otros niños en los hogares, y a percepciones de ella como espacio de estabilidad y refugio entre niños (factor de atracción), o de violencia, peligro y victimización (factor de expulsión). La relación con la calle es por lo tanto evolutiva y se presenta de forma regular como una alternativa a los niños y jóvenes como espacio de supervivencia material y afectivo, intercalado entre la familia y las instituciones de apoyo. La calle debe ser entendida pues como espacio de socialización entre pares (para encontrarse, comer y beber) y de recursos económicos por medio de un trabajo y del aprendizaje de competencias para ganar dinero (Cavagnoud, 2012) como el lustrado de botas, el lavado de parabrisas o el robo. Es un centro de gravedad variable, que se ofrece de manera inmediata a los niños para desplegar estrategias de trabajo y encontrar un marco de vida diferente a aquellos socialmente legitimados como la familia, la escuela y el medio institucional. De hecho, el significado y el uso de la calle divergen entre las secuencias T2 y T3. En la secuencia T2, la calle les da a los niños un estatus, una identidad construida en su grupo y un espacio de adquisición de habilidades que utilizan en acciones que involucran su vida cotidiana. En la secuencia T3, la calle es un espacio de vida momentáneo en alternancia con los hogares, el trabajo, el robo y la familia. Es un espacio que permite escaparse de la violencia doméstica cuando los niños retornan en su familia o de la violencia y reglas impuestas por los educadores en los centros de acogida. La calle releva entonces dos conjuntos de representaciones opuestas según el punto de vista de los niños y el de las instituciones; para los primeros es vista como un espacio de libertad y refugio para arreglárselas mientras que para los segundos, en particular los educadores, es percibida como un obstáculo a la formación moral del niño (Cavagnoud, 2014; Lucchini, 1993: 17). Este aspecto es fundamental pues permite dar cuenta de las dificultades de intervención de los programas de apoyo social a fin de integrar de forma duradera a los niños en situación de calle, tanto en el caso de La Paz 
y El Alto como en otras metrópolis. La fragmentación que caracteriza el trayecto vital de los niños y jóvenes, su aspiración a un ideal de autonomía y su deseo de reconocimiento como sujetos tienden también a debilitar las capacidades de intervención política.

\section{2. Hiato en torno a las necesidades de protección}

En La Paz y El Alto, más de diez asociaciones y O rganizaciones No Gubernamentales trabajan con niños que han huido de su familia. Los jóvenes en situación de calle están designados por la mayoría de estas instituciones como los «niños de las calles» 0 «de la calle» (street children en inglés) aunque su pertenencia a la calle y su presencia continua en este espacio no sean efectivas, como lo indica el caso de Wilson y otros jóvenes. Estas organizaciones proponen una variedad de actividades y serviciossegún una modalidad de acogida que puede ser a la vez abierta (unashoras durante el día) o cerrada (presencia continua en la institución). O rganizan acciones en los sectores de la alimentación, del cuidado, del higiene, de la instrucción y de la formación profesional subrayando hacia los niños valores morales de respeto de las reglas de vida fuera de la calle, la cual simboliza el desorden (Cavagnoud, 2014). Estas asociaciones y O NG implementan actividades lúdicas, deportivas y educativas presentándose como responsables de la reintegración de los niños en los espacios y roles reconocidos como socialmente legítimos.

No obstante, las instituciones que ofrecen una acogida de forma continua se enfrentan con la importante movilidad de los niños y experimentan mayores dificultades para mantenerlos en su estructura para una intervención a largo plazo. Las aspiraciones individuales a la autonomía invitan a los niños y jóvenes a tomar a cargo su destino y realizarse como sujetos construyendo un sentido a su propia existencia, la cual induce en este contexto de discontinuidad una circulación entre los diferentes espacios de socialización (grupo de pares, trabajo o robo en la calle, familia, servicio militar) ofreciendo formas específicas de protección en oposición a aquellas desarrolladas por los educadores y hogares de asistencia. Las elecciones individuales de los niños, sus deseos y proyectos se inscriben en un contexto de coacciones socioeconómicas, familiares e institucionales que intentan superar apoyándose en todos los soportes a su disposición y en la red de entornos que logran construir a lo largo de su trayecto dentro y alrededor de la calle. Esta les da también una protección más asequible y útil en caso de necesidades inmediatas 0 en el largo plazo. A pesar de condiciones materiales muy precarias, estos niños y jóvenes tratan de conservar un margen de maniobra que les ayuda a variar sus relaciones con los distintos espacios de socialización e inscribirse de esta manera en un proceso de subjetivación. Por tanto, el medio institucional se enfrenta con niños que pueden involucrarse en un campo social y paralelamente ubicarse a distancia de otros campos, 0 incluso implicarse en una forma de ruptura y bifurcación biográfica por la elección de otro modo de vida como el de la migración hacia otra provincia del país donde existen opciones laborales (por ejemplo a los Yungas para cosechar frutas u hojas de coca). Su lógica 
de acción sigue entonces sus estrategias de movilidad en la red de sociabilidad y de acuerdo a las oportunidades y a la evolución de las limitaciones que se les imponen en los diferentes campos de la vida cotidiana.

El ideal de autonomía que acompaña la modernidad y atraviesa sociedades anteriormente tradicionales, en particular en ciudades como La Paz y El Alto, contribuye a debilitar las posibilidades de intervención políticas de las ONG y del sector asociativo en general, confrontados con niños que ya no pertenecen, al menos simbólicamente, a la infancia como categoría jurídica y moral. Por lo tanto, se manifiesta una preferencia por el uso de servicios y programas sociales cuando resultan necesarios y responden a elecciones de socialización y a recomposiciones circunstanciales para enfrentar la precaridad en su dimensión estructural dominante y afirmar un rol y una identidad personal propia fuera de toda forma de dependencia que no corresponden con aquel ideal de autonomía.

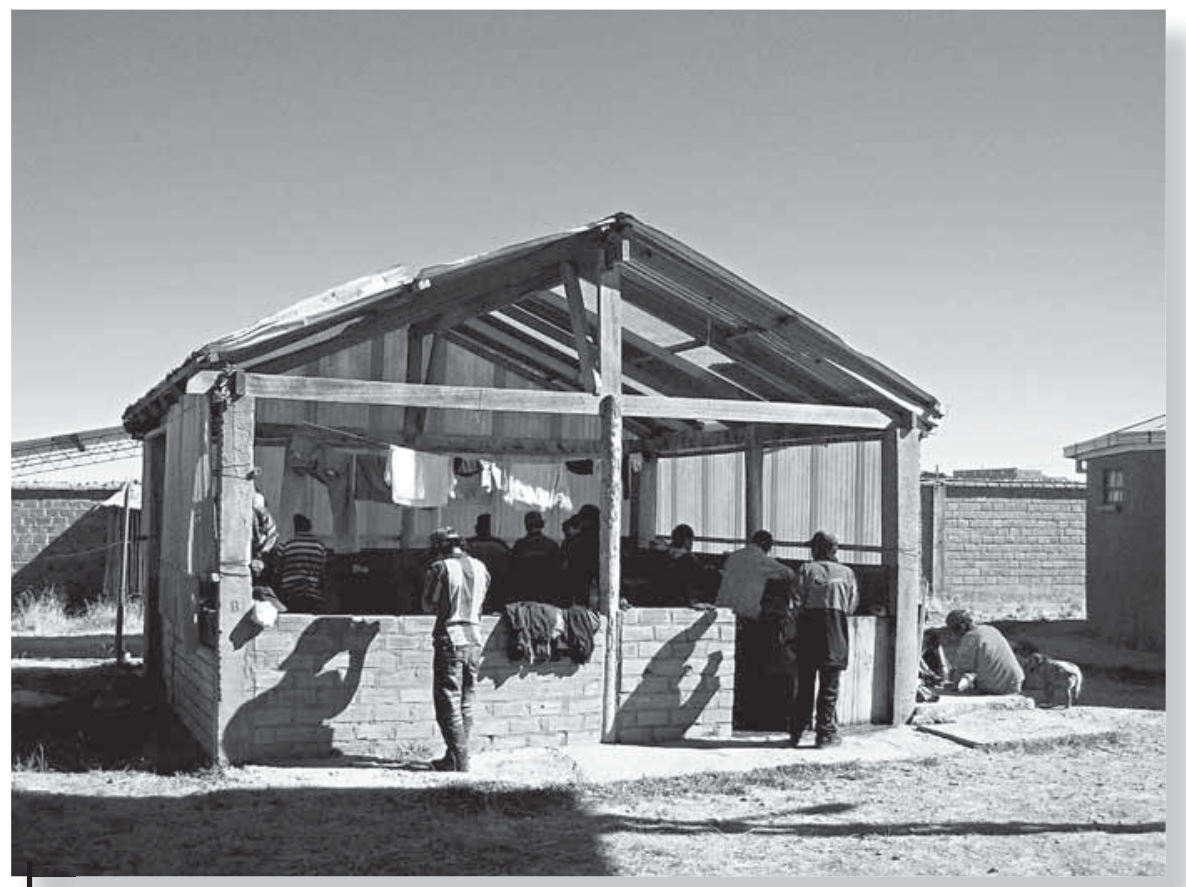

Figura 3 - Jóvenes lavando ropa en el centro Luz de Esperanza en El Alto (c) RC, $25 / 06 / 2011$

\section{CONCLUSIÓN}

La infancia en el contexto de la calle en La Paz y El Alto refleja prácticas y representaciones que se oponen claramente al paradigma de la Convención Internacional de los Derechos del Niño (CIDN) de 1989 así como de las 
intervenciones de las ONG y del medio asociativo. Remite a una dimensión constructivista de la infancia (James \& Prout, 1997), ilustrada por una heterogeneidad en la experiencia y manera de ser niño relacionadas con el grado de autonomía hacia la familia y los miembros de su entorno, y sus tomas de decisión así como a formas de participación social y prácticas alejadas del estatus de niño escolarizado, acompañado por los adultos en el marco de una protección instituida. Esta manifestación de la «pluralidad de la infancia» (Jenks, 1996) hace eco a las estructuras sociales en relación con el origen familiar y la historia personal de estos niños, su clase social y el contexto dominante de precariedad. Refleja un hiato con la concepción universal y el modelo de infancia formalizado por la CIDN que buscan aplicar las ONG y las instituciones de apoyo. Los niños y jóvenes en situación de calle encarnan una evolución hacia un estatus de niño autónomo, a diferencia de los principios de protección social de la CIDN que acompaña simultáneamente una individuación de los derechos y una forma de autonomía de los niños hacia el mundo adulto. La CID N subraya el principio de participación de los niños en la vida social. Les reconoce también un estatus de actores ante su propio porvenir incentivando su adquisición de una mayor autonomía que puede enfrentarse, como en los casos estudiados, con el cumplimiento de una mejor protección social de la infancia. Esta designa a la vez el discurso y las medidas a tomar por diferentes instituciones (M inisterios, O rganizaciones Internacionales y No Gubernamentales) para intervenir en las situaciones identificadas como formas de abuso, explotación o violencia contra los niños. Sin embargo, la protección de la infancia se puede entender también a partir de las prácticas de cuidado y educación desarrolladas por los mismosniños y su entorno inmediato en el contexto de la calle o fuera de ella. En este sentido, una forma de protección «cercana» que remite a las solidaridades interindividuales puede entrar en una relación de tensión con la protección «social» implementada a nivel político e institucional. El caso de los niños y jóvenes en situación de calle demuestra por tanto que la noción de protección en el sentido amplio no se adecúa sistemáticamente a los derechos del niño, a fortiori al abordar la cuestión del trabajo de los niños y del cuidado que se puede observar entre ellos en el espacio público o en el marco de los hogares de acogida.

Por último, la investigación llevada en La Paz y El Alto ha mostrado cómo los trayectos de los niños y jóvenes están entrecortados por secuencias entre la calle, los centros, retornos en la familia y la cárcel para la mayoría de ellos. En la misma vena, un número mayoritario de niños y jóvenes prefieren invertir una cantidad cotidiana de unos bolivianos para tener un cuarto básico en un hostal popular más que de dormir en la calle. Estos elementos indican cuánto la categoría de «niños de las calles» plantea un problema desde el punto de vista empírico, y conduce a una desaparición sui generis del fenómeno, considerando la realidad de estos niños para quienes la calle es solo un espacio entre otros, cada vez más efímero, que ocupa su vida cotidiana. 


\section{Referencias citadas}

BESSIN, M., BIDART, C. \& GRO SSETTI, M . (eds.), 2010 - Bifurcations. Les sciences sociales face aux ruptures et à l'événement, 402 pp.; París: La Découverte.

CASTEL, R. \& HARO CHE, C., 2001 - Propriété privée, propriété sociale, propriété de soi, 209 pp.; París: Hachette.

CAVAgNOUD, R., 2012 - L'enfance entre école et travail au Pérou. Enquête sur des adolescents à Lima, 248 pp.; París: Karthala.

CAVAGNOUD, R., 2014 - Enjeux moraux et discussion autour de la figure d'enfants des rues en Bolivie. Autrepart, 72: 165-181.

CAVAGNO UD, R., en prensa (a) - Infancia, calle y supervivencia. El caso de La Paz y El Alto (Bolivia). Debates en Sociología, 41.

CAVAGNOUD, R., en prensa (b) - Comprendre la notion de survie au fil des parcours d'enfants en situation de rue à La Paz et El Alto (Bolivie). In: Production politique et institutionnelle de l'enfance autour du monde (V. Pache, É. Guillermet \& C.-É. de Suremain, eds.); Liège: Presses Universitaires de Liège.

ELIAS, N., 1987 - La société des individus, 301 pp.; París: Fayard.

GO LAZ, V., 2009 - Mémoire, appréciation de la vie et entourage familial. In: Mémoire et démographie : regards croisés au Sud et au Nord (V. Golaz \& É. Lelièvre, eds.): 155167; Laval: Presses de I'U niversité Laval.

HÉLARD OT, V., 2010 - Vouloir ce qui arrive ? Les bifurcations biographiques entre logiques structurelles et choix individuels. In: Bifurcations. Les sciences sociales face aux ruptures et à l'événement (M. Bessin, C. Bidart \& M. Grossetti, eds.): 160-167; París: La Découverte.

INSTITUTO NACIO NAL DE ESTADÍSTICA (INE), 2011 - Encuesta Nacional de Hogares 2011; La Paz: INE.

INSTITUTO NACIO NAL DE ESTADÍSTICA (INE), 2013 - Censo Nacional de Población y Vivienda 2001 y 2012; La Paz: INE.

JAMES, A. \& PROUT, A. (eds.), 1997 - Constructing and Reconstruting Childhood. Contemporary Issues in the Sociological Study of Childhood, 280 pp.; Londres: Routledge.

JENKS, C., 1996 - Childhood, 192 pp.; Londres: Routledge.

LAZAR, S., 2013 - El Alto, ciudad rebelde, 297 pp.; La Paz: Plural Ed.

LELIĖVRE, É. \& VIVIER, G., 2001 - Évaluation d'une collecte à la croisée du quantitatif et du qualitatif. L'enquête Biographies et entourage. Population, 56 (6): 1043-1073.

LUCCHINI, R., 1993 - Enfants de la rue, identité, sociabilité, drogue, 272 pp.; París y Ginebra: Droz.

LU CCHINI, R., 1996 - Sociologie de la survie : I'enfant dans la rue, 336 pp.; París: Presses universitaires de France (PUF).

MARTU CCELLI, D., 2002 - Grammaires de l'individu, 712 pp.; París: Gallimard.

MÉRIENNE SIERRA, M., 1994 - Violence et tendresse. Les enfants de la rue à Bogotá, 100 pp.; París: L'H armattan.

MOLLERICO NA, J. Y., CO PA, J. \& CADENA, M. L., 2011 - «Paradorcito eres, ¿no ?» Radiografía de la violencia escolar en La Paz y El Alto, 142 pp.; La Paz: Fundación PIEB. 
La Paz y El Alto: discontinuidad y autonomización de los trayectos de los niños y jóvenes en situación de calle

NÉGRONI, C., 2010 - Ingrédients des bifurcations professionnelles : latence et événements déclencheurs. In: Bifurcations. Les sciences sociales face aux ruptures et à l'événement (M. Bessin, C. Bidart \& M. Grossetti, eds.): 176-183; París: La Découverte.

PÉREZ LÓ PEZ, R., 2009 - Vivre et survivre à M exico. Enfants et jeunes de la rue, 345 pp.; París: Karthala.

POU PEAU, F., 2009 - Les frontières de la métropolisation. Inégalités sociospatiales d'accès à l'eau et indicateurs de pauvreté à La Paz, Bolivie. Transcontinentales, 7: 81-104.

POU PEAU, F., 2011 - L'envers de la «ville aymara». M igration rurale, mobilité intraurbaine et mobilisations politiques à El Alto (Bolivie). Revue d'Études en Agriculture et Environnement, 92 (3): 417-440.

POUPEAU, F., 2013 - Enquêter sur les inégalités urbaines en Bolivie. Accès au terrain, positionnement du chercheur et construction d'objet. Genèses, 90 (1): 62-84.

PROGRAMA DE LAS NACIONES UNIDAS PARA EL DESARROLLO (PNUD), 2005 Informe sobre desarrollo humano en Bolivia; La Paz: PNUD.

SUREM AIN, C.-É. de, 2006 - Affinité horizontale et stratégies de survie parmi les 'enfants de la rue'. La bande Solitarios à La Paz (Bolivie). Revue Tiers Monde, 185: 109-128.

SUREM AIN, C.-É. de, 2013 - Des corps à la rue. Petite histoire d'une recherche-action participative auprès d'une bande d'enfants de la rue à La Paz (Bolivie). Corps, 11: 213-224.

TARACENA, E. \& TAVERA, M. L., 2005 - La fonction du groupe chez les enfants des rues à México. In : Contribution socio-anthropologique à l'enfant en grande difficulté dans I'espace public (S. Tessier, ed.): 13-38; París: L'H armattan.

TASSI, N., MED EIROS, C., RO DRÍGU EZ-CARM O N A, A. \& FERRU FIN O , G., 2013 - «H acer plata sin plata». El desborde de los comerciantes populares en Bolivia, 286 pp.; La Paz: Fundación PIEB.

YAPU, M. \& QUISBERT, M., 2008 - Jóvenes aymaras, sus movimientos, demandas y políticas públicas, $x+117$ pp.; La Paz: Fundación PIEB. 